\title{
LENGUA Y DERECHO: PERSPECTIVAS DE ESTUDIO
}

\author{
MAPI BALLESTEROS PANIZO \\ Universidad Internacional de la Rioja \\ mariapilar.ballesteros@unir.net \\ ANNA LÓPEZ SAMANIEGO \\ Universitat de Barcelona \\ alopezsam@ub.edu
}

\section{RESUMEN}

El derecho es una actividad profesional esencialmente lingüística. Desde los años 80 han proliferado estudios que han analizado las relaciones entre lingüística y derecho desde perspectivas diversas. El presente documento revisa las principales áreas de investigación que se enmarcan en este vasto campo de estudio y presenta los artículos que conforman este volumen: cinco trabajos que constituyen dos bloques temáticos netamente diferenciados. El primero presenta dos muestras de cómo la lingüística puede ponerse al servicio de la resolución de problemas jurídicos; el segundo ofrece la perspectiva del jurista sobre cómo un mejor conocimiento del lenguaje e incluso de otros lenguajes ajenos al derecho puede aplicarse a la mejora de la comunicación en el ámbito judicial.

PALABRAS CLAVE: lenguaje y derecho, discurso jurídico, formación del jurista, lingüística forense.

\section{LANGUAGE AND LAW: PERSPECTIVES OF STUDY}

\section{ABSTRACT}

Law is an essentially linguistic professional activity. Since the 1980s studies based on the analysis of the relationships between linguistics and law from diverse perspectives have proliferated. This document accounts for some of the main areas of research included in this vast field of study and presents the articles contained in this volume: five papers that are divided into two clearly differentiated thematic blocks. The first group of studies presents two examples of how linguistics can be used to solve legal problems; the second group of articles offers the jurist's perspective on how a better knowledge of language, even other languages outside the domain of law, can be applied to the improvement of communication in the judicial sphere.

KEYWORDS: Language and law, Legal discourse, Formation of the Jurist, Forensic linguistics.

\section{LAS RELACIONES ENTRE LENGUA Y DERECHO: PERSPECTIVAS DE ESTUDIO}

Si algo caracteriza al ingente número de publicaciones que han abordado en las últimas cuatro décadas la complejidad de las relaciones existentes entre lengua y derecho es su diversidad. El derecho es una actividad profesional esencialmente lingüística y de ello son conscientes tanto los juristas como los lingüistas que se han aproximado al estudio de la intersección entre estas disciplinas desde distintas perspectivas teóricas y aplicadas. Tal ha sido la relevancia y cantidad de estudios elaborados en esta línea que ha dado lugar a una macrodisciplina a la 
que los autores han atribuido denominaciones tan diversas como Lingüística Jurídica (Cornu 1990), Lingüística Forense (Gibbons 2004) o Lingüística Legal (Alcaraz 2005). Aunque de todas ellas probablemente es la primera, Lingüística Jurídica, la más clara y unívoca, Lingüística Forense parece ser la denominación que se ha impuesto en el ámbito internacional para referirse al conjunto de estudios que han analizado las relaciones entre lengua y derecho, especialmente desde los años 80 del siglo pasado.

Pese a la dificultad que entraña dar cuenta de este vasto campo de estudio, lo cierto es que las múltiples clasificaciones existentes de los ámbitos de investigación que pueden distinguirse dentro de la Lingüística Jurídica (Levi 1990, Borja Albí 2000, Gibbons 2004, Taranilla 2012: 22 y ss., entre otras) coinciden, a grandes rasgos, en identificar tres ámbitos de interés principales, que pueden describirse como sigue:

(1) El lenguaje como herramienta fundamental del Derecho, analizado, por lo general, desde una perspectiva teórica.

(2) El lenguaje como transmisor y perpetuador de relaciones de poder, desde una perspectiva crítica y sociológica.

(3) El lenguaje como prueba o como motivo de controversia en el ámbito judicial, desde un enfoque aplicado.

La primera de estas áreas de estudio es, hasta el momento, la más fructífera, y se encuentra en los orígenes del estudio de las relaciones entre lengua y derecho, que suelen vincularse a la publicación del trabajo de Mellinkoff (1963) sobre el discurso jurídico en inglés. Este ámbito de estudio, el más teórico y descriptivo de los tres, se centra en la caracterización del discurso jurídico y la descripción del papel que desempeña en la comunicación en ámbito judicial, a menudo desde una perspectiva crítica que suele llevar a denunciar los problemas de comunicación que a menudo comporta el llamado «dilema de la doble audiencia» (Gibbons 2004) que caracteriza a este discurso, dirigido tanto a especialistas en derecho como a los ciudadanos afectados, legos en la materia. En esta línea de investigación se enmarcan los trabajos fundacionales de autores como Lawrence Solan, Peter M. Tiersma o John Gibbons, en el ámbito anglosajón, y autores como Carles Duarte, desde la lingüística, o Cesáreo Rodríguez Aguilera, desde el derecho, para el ámbito del español. ${ }^{1}$

Tras la publicación de los primeros trabajos en esta línea, con el cambio de siglo la bibliografía se ha centrado, por un lado, en la identificación y caracterización de los rasgos lingüístico-discursivos que distancian al lenguaje jurídico de la lengua general, desde una perspectiva crítica que aboga por la

\footnotetext{
${ }^{1}$ Dado el carácter generalista de esta introducción, se citan únicamente algunos de los autores más influyentes de cada corriente. Para una revisión exhaustiva y detallada de la bibliografía existente sobre las relaciones entre lengua y derecho, puede consultarse Taranilla (2012: 19 y ss.).
} 
modernización y optimización de este lenguaje. La necesidad de que el ejercicio del derecho se realice mediante un lenguaje más claro ha sido defendida para el español tanto por lingüistas (de Miguel 2000, Montolío y López Samaniego 2008, Montolío et al. 2011, Jiménez Yáñez 2016) como por algunos juristas (Prieto de Pedro 1991, Bayo Delgado 1997, Cazorla 2007). Recientemente, la estrecha colaboración entre especialistas de ambas disciplinas ha dado lugar a publicaciones como el Libro de estilo de la Justicia (Muñoz Machado (dir.) 2017).

Por otro lado, aún en esta misma primera área de análisis del discurso jurídico, ha resultado también especialmente prolífico, sobre todo en el ámbito anglosajón, el análisis del lenguaje como herramienta comunicativa en los distintos eventos que conforman la práctica del derecho, concretado en el análisis de géneros jurídicos como la sentencia judicial, las instrucciones al jurado (especialmente en el ámbito anglosajón), el interrogatorio oral o la interacción oral o escrita entre abogado y cliente, así como del lenguaje propio de leyes y contratos. Todavía dentro de esta línea, más recientemente los lingüistas han mostrado especial interés por analizar la construcción de las principales tipologías textuales relevantes en el discurso jurídico; a saber: la argumentación jurídica (que ya había sido objeto de análisis del derecho y de los estudios de retórica y lógica formal desde la segunda mitad del siglo XX), con trabajos como los de Davide Mazzi sobre el inglés (cfr. 2007); y la narración, que permite analizar cómo se construye la versión probada de los hechos encausados mediante los distintos documentos que se suceden a lo largo de los procesos judiciales. Esta última línea ha sido abordada en español por Raquel Taranilla en múltiples publicaciones (cfr. 2012).

Pueden englobarse también en esta línea de análisis del lenguaje como herramienta al servicio de los juristas algunos trabajos recientes que analizan el uso del lenguaje jurídico en contextos multilingües, como la Unión Europea o las prácticas de arbitraje, llevados a cabo desde una perspectiva contrastiva (Ondelli 2013, Bhatia et al. 2014, Tessuto et al. 2016), así como desde los estudios de traducción e interpretación, en el que destacan, en español, los trabajos desarrollados por Enrique Alcaraz.

Por lo que respecta a la segunda de las áreas de investigación en lengua y derecho mencionadas, el análisis del discurso jurídico como mecanismo para reproducir, legitimar y perpetuar las relaciones asimétricas de poder existentes entre los distintos participantes en un juicio o bien entre el sistema jurídico y el ciudadano en general, esta ha sido especialmente abordada hasta el momento en el ámbito anglosajón, en trabajos como los de Conley y O’Barr (2005²) o Stygall (2012). Sin embargo, esta línea de investigación ha sido aún muy poco transitada en el ámbito hispánico, más allá de la relevancia que han cobrado en la última década los estudios que defienden la necesidad de modernizar el discurso jurídico (mencionados ya en relación con el enfoque anterior), que entroncan también claramente con este enfoque crítico sobre el discurso jurídico, por cuanto ponen de relieve la distancia existente entre el lenguaje empleado por los agentes 
jurídicos y el uso general de la lengua por parte de la ciudadanía. Este movimiento en defensa de un discurso jurídico claro ha calado en múltiples países y tradiciones jurídicas y ha sido abordado en español, especialmente, por Estrella Montolío y el grupo EDAP.

La última área de investigación mencionada se identifica con la Lingüística Forense en su definición más restrictiva, que se ocupa de emplear el conocimiento experto de la lengua como prueba ante los tribunales en la resolución de delitos lingüísticos o con base lingüística, como el perjurio, la difamación o el plagio, así como en la resolución de problemas de interpretación de leyes o contratos. De obligada cita en este ámbito son nombres como los de Malcolm Coulthard, Robert Shuy, John Gibbons y, en español, Ma Teresa Turell, fundadora del ForensicLab, laboratorio de Lingüística Forense del Instituto Universitario de Lingüística Aplicada (IULA).

El desarrollo de las relaciones entre lengua y derecho que se ha presentado escuetamente hasta aquí ha cristalizado en los últimos años en el surgimiento de puntos de encuentro para los especialistas como asociaciones (Asociación Internacional de Lingüistas Forenses), revistas (International Journal of Speech, Language and the Law) o incluso cursos de formación en comunicación jurídica dirigidos a profesionales. Precisamente uno de estos cursos, el III Curso de comunicación para juristas, celebrado en julio de 2016 en la Universidad Internacional Menéndez Pelayo, constituye el punto de partida de este volumen monográfico, que reúne algunas de las aportaciones presentadas en él. El volumen comprende trabajos elaborados por lingüistas y juristas, que abordan distintos aspectos de las relaciones entre lengua y derecho que pueden englobarse en las tres perspectivas de análisis mencionadas en esta introducción.

\section{CONTENIDO DEL VOLUMEN}

El volumen consta de dos bloques temáticos netamente diferenciados: el primero, que contiene los dos primeros artículos, presenta dos muestras de cómo la lingüística puede ponerse al servicio de la resolución de problemas jurídicos; en tanto que el segundo se compone de tres artículos que ofrecen la perspectiva del jurista sobre cómo un mejor conocimiento del lenguaje e incluso de otros lenguajes ajenos al derecho puede aplicarse a la mejora de la comunicación en el ámbito judicial.

El primer bloque, que aborda la cuestión del lenguaje jurídico desde una perspectiva filológica, subraya que la naturaleza específica del lenguaje del Derecho puede terminar provocando problemas jurídicos de proporciones destacables. Se abre con el artículo de González Salgado, que muestra el modo en que la puntuación, correctamente empleada, es capaz de resolver los principales problemas de interpretación de los textos jurídicos. Porque puntuar es, precisamente, un sistema de estrategias destinadas a controlar la 
interpretación. Estos textos acostumbran a estar poco y mal puntuados, a contener oraciones subordinadas concatenadas que entorpecen la eficacia comunicativa de los textos cuando no causan importantes problemas. En su contribución Salgado compila, a partir de su experiencia profesional de diez años de asesoría lingüística en el despacho Uría Menéndez Abogados, las incorrecciones de puntuación que cometen más frecuentemente los abogados.

En particular el autor divide en tres clases los errores más frecuentes. La primera contempla los usos incorrectos de la coma; la segunda se destina a otros signos de puntuación como el punto, punto y coma, dos puntos y puntos suspensivos. Este segundo bloque es menos problemático, en lo que a interpretación se refiere; pero contiene transgresiones de la norma ortográfica que también pueden afectar negativamente a la imagen del escritor. La tercera clase de incorrección se refiere a los signos utilizados para la delimitación de incisos en los textos jurídicos.

Por su parte, Hidalgo Navarro aborda el problema de la ambigüedad en los textos jurídicos, desde la perspectiva de la Lingüística Forense. Como demuestra el autor, la ambigüedad en algunos textos jurídicos puede ser causa de controversia lingüística e, incluso, motivo de pleito legal. La Lingüística Forense aplica una gran diversidad de disciplinas lingüísticas, según el aspecto considerado a la hora de reconocer en el producto lingüístico elementos de evidencia probatoria. Mediante análisis grafémicos, fonéticos, morfológicos, sintácticos, semántico-léxicos, pragmáticos, sociolingüísticos o estilométricos, la lingüística forense puede demostrar su utilidad para la presentación de evidencias probatorias diversas.

A partir de un caso práctico de ambigüedad, Hidalgo ofrece una serie de pautas lingüísticas (ortográficas, morfosintácticas, léxico-semánticas, discursivotextuales, etc.) capaces de facilitar su resolución. En concreto, parte de un diagnóstico que explica las causas por las que surgen los problemas de ambigüedad, determinando operativamente su tipología y ofrece, además, orientaciones útiles para superar las dificultades que ocasionan.

El segundo bloque del volumen constituye una mirada jurídica al problema del lenguaje y aborda dos cuestiones capitales; a saber: la adopción del lenguaje científico por parte del Derecho y la formación de los juristas en comunicación. Hernández Galilea se ocupa de del primero de estos aspectos. A partir de la constatación de que, en los últimos tiempos, han proliferado las referencias técnicas extrajurídicas y la presencia de expertos -científicos o técnicos- en los juicios, el autor explica el modo en que la penetración de la ciencia en el ámbito del derecho ha llegado a poner en tela de juicio la idoneidad del sistema judicial actual. En efecto, el prestigio del que goza la ciencia va unido a la tentación de pretender que sean las ciencias naturales, las ciencias experimentales y la tecnología las que marquen el rumbo del progreso social.

El autor plantea la necesidad de situar el problema en el contexto de la comunicación desde la perspectiva de la pragmática para superar la visión que 
denomina tecnocrática y volver a recuperar el sentido genuino de la función judicial. El enfoque pragmático que propone Hernández Galilea permite clarificar la posición del perito y del juez, así como desplegar las normas procesales en todo su potencial:

\begin{abstract}
Al poner en primer plano el aspecto comunicativo de la prueba pericial, en lugar del epistemológico, no sólo se clarifica la relación entre ciencia y el derecho, entre el juez y el perito, sino que, al mismo tiempo, se conjura la presión tecnocrática que, cuando exige al juez unos conocimientos determinados, está, en el fondo, colonizando la función jurisdiccional en aquellos ámbitos, cada vez más, en los que la presencia de la ciencia o la tecnología se hace sentir con más fuerza. (p. 122).
\end{abstract}

Las dos últimas contribuciones reflexionan sobre la formación de los juristas. En el artículo titulado La literatura en la formación de jueces y abogados, Arsuaga señala los riegos que se derivan de la adopción acrítica del lenguaje científico por parte del Derecho (Nussbaum 1995). Tal actitud es positivista y se debe a la tendencia que existe de aislar el Derecho de todo contexto y se materializa en el tipo de retórica que se utiliza en las diferentes democracias. Además, se trata de una actitud que explica actuaciones terribles de los juristas, en la historia reciente. En el trabajo de Arsuaga se desgranan las reflexiones que White y Weisberg han expuesto en diferentes trabajos y se llama la atención sobre la necesidad de acudir a la formación literaria de los juristas como método para rectificar el rumbo que está tomando el Derecho.

Para la autora, la literatura proporciona «madurez intelectual, capacidad crítica cultural, el dominio de ciertas habilidades literarias, así como una cierta calidad en el pensamiento y la expresión» (1995: 131), habilidades que son fundamentales para los juristas, pues su tarea consiste en regular las relaciones interpersonales a través de un conjunto de proposiciones lingüísticas. En efecto, el Derecho es lenguaje, aunque no se agota en el lenguaje. Por eso es necesario que los juristas no se queden atrapados en los límites del significado literal de las palabras, sino que han de ser capaces de contextualizar y de nutrirse de otras áreas del conocimiento humano. Para esta autora, la literatura no es un complemento de la formación del jurista, sino un constituyente esencial que debería estar en la base de su preparación. Solo así podrán los profesionales del derecho actuar con criterio en las situaciones más diversas, ya que el mundo en que se mueve el jurista es el mundo social, y no el técnico.

Por último, Carretero expone las iniciativas públicas y privadas orientadas a la formación lingüística de los juristas. Asimismo, la autora recoge una lista de los errores comunes que cometen los juristas e incluye algunas propuestas de mejora.

Si bien la mayoría de los trabajos realizados en la interfaz entre lengua y derecho revisados en el apartado anterior están elaborados desde una perspectiva exclusivamente jurídica o únicamente lingüística, este monográfico se alinea con algunos de los más recientes, que abogan por la necesidad de una 
colaboración estrecha entre ambos especialistas. Solo desde una verdadera comunicación entre ambas disciplinas se podrá contribuir a mejorar la calidad de los textos jurídicos, a evitar o resolver satisfactoriamente los problemas jurídicos derivados de problemas comunicativos y a enriquecer la formación en comunicación de los futuros juristas.

\section{BIBLIOGRAFÍA}

ALCARAZ, E. (2005), «La lingüística legal: el uso, el abuso y la manipulación del lenguaje jurídico», en Lingüística forense, lengua y derecho. Conceptos, métodos y aplicaciones, Turell, M T. (ed.), Barcelona, Institut Universitari de Lingüística Aplicada, 49-66.

BAYO, J. (coord.) (1997), Lenguaje judicial, Madrid, CGPJ.

Bhatia, V.; GARZONE, G; SAlvi, R.; TEssutO, G. y Williams, C. (eds.) (2014), Language and Law in Professional Discourses: Issues and Perspectives, Cambridge, Cambridge Scholars Publishing.

BORJA, A. (2000), El texto jurídico inglés y su traducción al español, Barcelona, Ariel Derecho. CAZORLA, L. M. (2007), El lenguaje jurídico actual, Madrid, Aranzadi.

CONLEY, J. M. y W. M. O’ Barr (2005² [1998]), Just words. Law, Language and Power, ChicagoLondon, The University of Chicago Press.

CORNu, G. (1990), Linguistique juridique. París, Montchrestien.

DE Miguel, E. (2000), «El texto jurídico-administrativo: análisis de una orden ministerial», Revista de Lengua y Literatura Españolas, 2, 6-31.

GIBBONS, J. (2004), Forensic linguistics. An introduction to language in the justice system, Oxford, Blackwell Publishing.

JiMÉNEZ YÁÑEZ, R. Mạ (2016), Escribir bien es de justicia, Cizur Menor (Navarra), Aranzadi.

LEVI, J. N. (1990), «The study of language in the judicial process» en Language in the judicial process, Levi, J. N. y Walker, A. G. (eds.), London-New York, Plenum Press, 3-35.

MAZZI, D. (2007), The Linguistic Study of Judicial Argumentation, Modena, Edizioni Il Fiorino.

MelLiNKOFF, D. (1963), The Language of the Law, Boston-Toronto, Little \& Brown Company.

Montolío, E. (dir.); GARcía ASEnSiO, Ma Á.; Gras, P.; LÓPEZ SAMANieGO, A.; POlAnCO, F.; TARANILLA, R. y YÚFERA, I. (2011), Estudio de campo. Lenguaje escrito. Comisión para la modernización del lenguaje jurídico, Madrid, Ministerio de Justicia.

MONTOLÍO, E. y LÓPEZ SAMANIEGO, A. (2008), «La escritura en el quehacer judicial: la propuesta aplicada en la Escuela Judicial de España», Revista Signos: estudios de lingüística, 66, 33-64.

MuÑOZ MACHADO, S. (dir.) (2017), Libro de estilo de la Justicia, Madrid, Espasa.

ONDELLI, S. (ed.) (2013), Realizzazioni testuali ibride in contesto europeo. Lingue dell'UE e lingue nazionali a confronto, Trieste, EUT Edizioni Università di Trieste.

PRIETO DE PEDRO, J. (1991), Lenguas, lenguaje y Derecho, Madrid, Civitas.

STYGALL, G. (2012), «Discourse in the US courtroom» en The Oxford Handbook of Language and Law, Tiersma, P. y Solan L. (eds.), Oxford, Oxford University Press, 369-380. 
TARANILLA, R. (2012), La Justicia Narrante: un estudio sobre el discurso de los hechos en el proceso penal, Cizur Menor (Navarra), Aranzadi.

Tessuto, G.; Bhatia, V.; Garzone, G; Salvi, R. y Williams, C. (eds.) (2016), Constructing Legal Discourses and Social Practices: Issues and Perspectives, Cambridge, Cambridge Scholars Publishing. 